\title{
ALTERAÇÕES FETAIS INDUZIDAS PELO USO DE ANTIINFLAMATÓRIOS DURANTE A GESTAÇÃO
}

\author{
FETAL ALTERATIONS INDUCED BY THE USE OF ANTIINFLAMMATORY \\ DRUGS DURING GESTATION
}

\author{
Cláudia Sampaio Fonseca ${ }^{1}$ Marlene Isabel Vargas Viloria ${ }^{2}$ Leandro Repetti $^{3}$
}

- REVISÃO BIBLIOGRÁFICA -

RESUMO

Em geral, todos os efeitos dos antiinflamatórios estão relacionados com a inibição da ciclo-oxigenase (COX) do ácido araquidônico e, portanto, inibição da produção de prostaglandinas e tromboxanos. Existem dois tipos de COX, quais sejam COX-1 e COX-2. A COX-1 é uma enzima constitucional expressa em muitos tecidos, incluindo plaquetas sangüíneas, $e$ está envolvida na homeostase tecidual. Por outro lado, a COX-2 é induzida em células inflamatórias quando elas são ativadas, sendo considerada a enzima que produz os mediadores da inflamação da classe dos prostanóides. A ação dos antiinflamatórios está relacionada à inibição da COX-2 e é provável que seus efeitos indesejados se devam principalmente à inibição da COX-1. Tratamentos maternos com antiinflamatórios não esteroidais (AINEs) têm sido associados, com freqüência, à vasoconstrição do ducto arterioso fetal, hipertensão arterial pulmonar e inibição da agregação plaquetária. Alterações na hemostasia são alguns dos efeitos colaterais produzidos pelo uso incontrolado dos AINEs, os quais induzem a um desequilíbrio na liberação de prostaglandinas e tromboxanos, que se reflete na adesividade e agregação plaquetária. As alterações hemostáticas observadas em neonatos, decorrentes do uso de salicilatos pela mãe, ocorrem devido à inibição da agregação plaquetária e à diminuição da atividade do fator XII relacionado à coagulação sangüínea. Estudos em camundongos revelaram que o uso de corticóides durante a gestação pode levar a anormalidades no desenvolvimento fetal, por alterações na diferenciação celular.

Palavras-chave: antiinflamatórios, gestação, alterações fetais.

\section{SUMMARY}

In general, all effects of the antiinflammatory drugs are related to the inhibition of the arachidonic acid and inhibition of prostaglandins and tromboxans production. Two types of ciclooxygenasis (COX) exist, which are COX-1 and COX-2. COX-1 is a constitutional enzyme expressed in many sites, including platelets, and is involved in tecidual homeostasis. On the other hand, COX-2 is induced in inflammatory cells when they are activated, and it is considered as being the enzyme that produces the mediators of the inflammation. The action of antiinflammatory drugs is related to the inhibition of $\mathrm{COX}-2$ and is probable that their undesirable effects are due mainly to the inhibition of COX1. Maternal treatments with NSAIDs have been frequently associated, with the vasoconstriction of the fetal ductus arteriosus, lung arterial hypertension and inhibition of platelet aggregation. Alterations in hemostasis are some of the collateral effects produced by the indiscriminate use of NSAIDs, which induce to an unbalance in the prostaglandins and tromboxans liberation, that are reflected in the adhesiveness and platelet aggregation. The haemostatic alterations observed in neonates, caused by salicilates's ingestion by the mother, are due to inhibition of the platelet aggregation and the decrease of the activity of the factor XII related to the clotting. Studies in mice revealed that corticoids's use during the gestation can lead to abnormalities in fetal development, because of the alterations in the cellular differentiation.

Keywords: antiinflammatory drugs, pregnancy, fetal alterations.

\footnotetext{
${ }^{1}$ Médico Veterinário, Doutorando em Medicina Veterinária, Área de Concentração Cirurgia Veterinária, pela Faculdade de Ciências Agrárias e Veterinárias, Universidade Estadual Paulista (UNESP), Campus de Jaboticabal. Rodovia Carlos Tonanni, Km 5, 14870-000, Jaboticabal, SP. Professor de Técnica Cirúrgica, Anestesiologia e Patologia Cirúrgica da Universidade de Marília. E-mail: cfonsecaca@bol.com.br. Autor para correspondência.

${ }^{2}$ Médico Veterinário, Professor Adjunto IV do Departamento de Veterinária da Universidade Federal de Viçosa, MG

${ }^{3}$ Médico Veterinário, Mestrando em Nutrição e Produção Animal pela UNESP, Campus de Botucatu, SP.
} Recebido para publicação em 18.12.00. Aprovado em 12.09.01 


\section{INTRODUÇÃO}

Os agentes antiinflamatórios agem no processo de redução da reação inflamatória por inibirem a liberação de prostaglandinas, atuando em etapas préestabelecidas na via do ácido araquidônico (GUYTON, 1992).

Os glicocorticóides constituem um grupo de drogas com efeitos antiinflamatórios e imunossupressores comumente utilizados em Medicina Veterinária. São agentes recomendados para o tratamento de doenças alérgicas e imunomediadas, choque séptico e cardiogênico e traumatismos do sistema nervoso central e da coluna vertebral (OSTENSEN, 1998). Outro grupo de drogas com efeitos antiinflamatórios diz respeito aos agentes antiinflamatórios não esteroidais, comumente referidos como AINEs. Seus maiores efeitos farmacológicos são redução do edema, eritema e dano tecidual resultante de processos inflamatórios (OSTENSEN, 1998).

Tratamentos maternos com AINEs têm sido associados, com freqüência, à vasoconstrição do ducto arterioso fetal, hipertensão arterial pulmonar e inibição da agregação plaquetária (HICKOK et al., 1989). Estudos sobre os efeitos dos antiinflamatórios demonstram alta incidência de síndrome de hipertensão pulmonar persistente no recém-nascido e de anormalidades na hemostasia (OSTENSEN, 1998).

A escassez de dados na literatura Médica Veterinária relativos aos efeitos destas drogas durante a gestação e suas implicações nos fetos justifica a importância desta revisão bibliográfica.

\section{PROSTAGLANDINAS E ANTIINFLAMATÓRIOS}

As prostaglandinas (PGs) constituem família complexa de derivados de ácidos graxos, sintetizadas por quase todas as células dos mamíferos. Possuem efeitos intracelulares importantes, sendo liberadas nos líquidos teciduais tanto em condições fisiológicas como patológicas (GUYTON, 1992; MILLER \& MARKS, 1994). A síntese de PGs ocorre quando as células são estimuladas a liberar o ácido araquidônico através da ativação dos receptores específicos ou ainda pela ativação inespecífica induzida pelo estresse mecânico (DAVIES \& MACINTYRE, 1992). Estímulos fisiológicos, farmacológicos e patológicos ativam a enzima fosfolipase $\mathrm{A}_{2}$, liberando ácido araquidônico dos fosfolipídeos celulares. O primeiro passo na síntese das PGs e dos tromboxanos (TXs) é mediado pela enzima ciclo-oxigenase (COX), a qual catalisa a incorporação de oxigênio ao ácido araquidônico com subseqüente formação de endoperóxidos cíclicos (ADAMS, 1992).

Sabe-se que a COX é o sítio de bloqueio dos AINEs, deste modo, tais drogas impedem a conversão do ácido araquidônico nos endoperóxidos cíclicos e, consequientemente, a formação de PGs e TXs é igualmente prejudicada. Entretanto, a aspirina inibe a COX de maneira irreversível sendo que seu efeito inibidor sobre a produção das PGs, a partir das plaquetas, persiste até que novas plaquetas sejam produzidas pela medula óssea (ADAMS, 1992; BAVOUX et al., 1992).

Existem dois tipos de COX, quais sejam COX-1 e COX-2. A COX-1 é uma enzima constitucional expressa em muitos tecidos, incluindo plaquetas sangüíneas, e está envolvida na homeostase tecidual. Por outro lado, a COX-2 é induzida em células inflamatórias quando elas são ativadas, e é considerada como sendo a enzima que produz os mediadores da inflamação da classe dos prostanóides. A ação dos antiinflamatórios está relacionada à inibição da COX-2 e é provável que seus efeitos indesejados se devam principalmente à inibição da COX-1 (DARMMANN, 1999; SIMON, 1999). Segundo SIMON (1999), a COX-1 é a forma constitutiva predominante da enzima e está expressa em toda parte do organismo, desempenhando determinadas funções homeostáticas, como proteção da mucosa gástrica, influência no fluxo sangüíneo renal e atuação na coagulação sangüínea, controlando a agregação plaquetária. Ao contrário, a COX-2 é a forma induzida da enzima, expressa em resposta ao estímulo inflamatório, estando envolvida na produção daquelas prostaglandinas que são mediadores da dor e suportam o processo inflamatório.

Os efeitos terapêuticos dos AINEs são o resultado da inibição da COX-2, enquanto os efeitos tóxicos se devem principalmente à inibição da COX1. Diferentes AINESs exibem potências diferentes contra a COX-1 em comparação à COX-2, o que explica a variação de efeitos colaterais dos AINEs (DARMMANN, 1999).

Por outro lado, os corticóides têm sido considerados como agentes inibidores da produção de prostaglandinas, pela ação inibitória que exercem sobre a fosfolipase $A_{2}$, por meio da liberação de lipocortina-1 (mediador protéico antiinflamatório). O resultado final da ação destes antiinflamatórios é a parcial ou total redução da liberação dos mediadores pró-inflamatórios. A lipocortina-1 atua por seqüestrar o substrato fosfolipídico e/ou inibir diretamente a enzima. Qualquer um desses mecanismos poderia contribuir para a redução na 
produção tanto do fator ativador de plaquetas quanto dos eicosanóides, observada na presença de corticóides (OSTENSEN, 1998; SIMON, 1999).

Os glicocorticóides exógenos inibem a expressão da COX-2, inibindo a transcrição do gene relevante, reduzindo a geração de prostanóides nas células inflamatórias, porém exercem pouco ou nenhum efeito sobre a enzima constitutiva COX-1 (SIMON, 1999).

\section{TOXICIDADE FETAL DOS ANTIINFLA- MATÓRIOS}

\section{Fechamento Prematuro do Ducto Arterioso Fetal}

O sistema circulatório fetal, por meio de arranjos anatômicos especiais, atua de modo diferente do adulto. O sangue que vem da placenta pela veia umbilical passa pelo ducto venoso, desviando-se do fígado. A seguir, a maior parte de sangue que entra no átrio direito, proveniente da veia cava, é dirigida através do forame oval para o átrio esquerdo. Deste modo, o sangue oxigenado proveniente da placenta entra para o lado esquerdo do coração, sendo bombeado pelo ventrículo esquerdo para os vasos da cabeça e membros superiores. O sangue que entra no átrio direito pela veia cava superior dirige-se, através da válvula tricúspide, para o ventrículo direito. Esse sangue, em sua maior parte desoxigenado, é bombeado pelo ventrículo direito para a artéria pulmonar e, a seguir, em grande parte, através do canal arterial, para a aorta descendente às duas artérias umbilicais, para a placenta, onde o sangue é oxigenado (GUYTON, 1992).

Ao nascimento, ocorrem alterações circulatórias que consistem na perda do elevado fluxo sangüíneo pela placenta, o que duplica a resistência vascular sistêmica, elevando a pressão aórtica, bem como a pressão no átrio e ventrículo esquerdos. Por outro lado, a resistência vascular pulmonar diminui em conseqüência da expansão dos pulmões (GUYTON, 1992).

O ducto arterioso também se fecha ao nascimento. $\mathrm{O}$ aumento da resistência sistêmica eleva a pressão aórtica, enquanto a diminuição da resistência pulmonar reduz a pressão arterial pulmonar. Em conseqüência, dentro de poucas horas após o nascimento, o sangue começa a fluir em sentido retrógrado, da aorta para a artéria pulmonar, através do ducto arterioso, em vez de seguir na direção oposta como ocorria na vida fetal. Todavia, depois de poucas horas, a parede muscular do ducto arterioso sofre contração acentuada e, dentro de poucos dias, a constrição é suficiente para interromper todo o fluxo sangüíneo (GUYTON,
1992). A causa do fechamento do ducto arterioso está relacionada ao aumento de oxigenação do sangue que flui através dele (GOOTMAN \& GOOTMAN, 1983).

Durante a gestação, observa-se que as prostaglandinas vasodilatadoras $\left(\begin{array}{llll}\mathrm{PGE}_{2} & \mathrm{e} & \mathrm{PGI}_{2}\end{array}\right)$ encontram-se em níveis elevados, enquanto $\mathrm{O}$ tromboxano $\mathrm{A}_{2}\left(\mathrm{TXA}_{2}\right)$, potente vasoconstritor, encontra-se em seu nível basal. Imediatamente após o nascimento, ocorre aumento súbito dos níveis de TXA $_{2}$ que, segundo REYES (1993), é o responsável pelo fechamento dos vasos umbilicais e do ducto arterioso.

Estudos experimentais sugerem que a prostaglandina $\mathrm{E}$ (PGE) e a prostaglandina $\mathrm{F}$ (PGF) estão envolvidas na regulação da resistência vascular do ducto arterioso. No útero, o bloqueio na produção das PGs tem produzido vasoconstrição ou fechamento do ducto arterioso em ovelhas e ratos (MANCHESTER $\boldsymbol{e} \boldsymbol{t} \boldsymbol{a l} .$, 1976). Segundo GORDON \& SAMUELS (1995), isto ocorre porque a prostaciclina $\left(\mathrm{PGI}_{2}\right)$ e a $\mathrm{PGE}_{2}$ são as responsáveis pela manutenção do ducto arterioso.

RUDOLPH (1981) demonstrou o fechamento do ducto arterioso fetal em ovelhas gestantes submetidas a tratamento com $30 \mathrm{mg} / \mathrm{kg}$ de aspirina, a cada 24 horas, durante três dias, no terço final de gestação. Ele relatou aumento significativo da pressão arterial pulmonar fetal, diminuição do consumo sangüíneo por meio do ducto arterioso e aumento das resistências no interior do ducto arterioso por vasoconstrição. O autor confirma que esta vasoconstrição é devida à inibição da síntese de $\mathrm{PGE}_{2}$. Assim, o fechamento prematuro do ducto arterioso pode resultar em falência cardíaca ao nascimento devido a alterações valvulares, tais como insuficiência da tricúspide (LOFFREDO $\boldsymbol{e t} \boldsymbol{a l}$., 1989).

BAVOUX (1992) relatou a importância de se realizar um estudo epidemiológico sobre os efeitos cardiopulmonares induzidos pelos AINEs quando utilizados durante a gestação. Segundo o autor, os efeitos cardiopulmonares destas drogas incluem fechamento prematuro do ducto arterioso, que pode ser importante para ocasionar hipertensão pulmonar pelo aumento da pressão arterial pulmonar, além de insuficiência da válvula tricúspide.

A este respeito, CABROL et al. (1987) concluíram que os antiinflamatórios promovem a vasoconstrição do ducto arterioso e aumentam a resistência vascular pulmonar, o que resulta em elevação da pressão ventricular direita, podendo causar isquemia subendocardial e calcificação no músculo papilar da válvula tricúspide e, consequientemente, insuficiência valvular. 
REIN et al. (1999) administraram diclofenaco, um AINEs, a uma mulher na $36^{\mathrm{a}}$ semana de gestação, pois a mesma queixava-se de fortes dores na região pélvica. Os autores observaram que o ducto arterioso do feto apresentava vasoconstrição com evidência de hipertensão ventricular direita, o que foi resolvido com a interrupção do tratamento.

\section{Alterações na Hemostasia}

Alterações na hemostasia são alguns dos efeitos colaterais produzidos pelo uso indiscriminado dos AINEs, os quais induzem a um desequilíbrio na liberação de prostaglandinas e tromboxanos, que se reflete na adesividade e agregação plaquetária (BAVOUX, 1992).

As alterações hemostáticas observadas em neonatos, decorrentes do uso de salicilatos pela mãe, ocorrem devido à inibição da agregação plaquetária e à diminuição da atividade do fator XII relacionado à coagulação sangüínea. A aspirina é rapidamente transferida da mãe ao feto, através da barreira placentária, e significantes concentrações do salicilato podem ser encontradas no neonato (DEKKER \& SIBAI, 1993).

Segundo SCHROR (1997), o efeito antitrombótico do ácido acetilsalicílico é devido à inibição da função plaquetária pela acetilação da ciclo-oxigenase plaquetária, inibindo a formação do TX de maneira irreversível. Como o ácido acetilsalicílico inibe somente a produção materna de TX, sem alterar a produção de $\mathrm{PGI}_{2}$, VIINIKA et al. (1993) recomendam cautela durante o uso em pacientes gestantes, para evitar alterações na hemostasia fetal.

STUART et al. (1982) observaram alterações hemostáticas somente em crianças recémnascidos cujas mães haviam ingerido salicilato 10 dias antes do parto previsto, e concluíram que os neonatos são mais susceptíveis aos efeitos da aspirina que as mães. Esta susceptibilidade pode ser devida a alterações nas plaquetas do neonato que apresentam anormalidades fisiológicas na adesividade, agregação e liberação de grânulos do citosol, prejudicando a função plaquetária.

Alterações hemorrágicas associadas à dose da aspirina nos últimos cinco dias de gestação foram relatadas por STUART et al. (1982), caracterizadas na mãe por hemorragia antes, durante e após o parto e por petéquias, hematomas e hemorragia subconjuntival nas crianças recémnascidas.

BAVOUX et al. (1992) relataram que a toxicidade de outros AINEs relacionados com a função plaquetária e o aparecimento de hemorragias é menos significativo e concerne apenas à aspirina.
STUART et al. (1982) observaram petéquias, púrpura e hematoma cefálico em crianças recém-nascidas cujas mães ingeriram salicilato durante a gestação; sendo que em um caso descrito, a criança teve que ser submetida à transfusão sangüínea devido à intensa hemorragia gastrointestinal. Demonstraram, ainda, que a ingestão de aspirina antes do parto está associada a alterações clínicas importantes no mecanismo hemostático do neonato, tendo sido observado equimoses, petéquias, hemorragia subconjuntival, hematomas cefálicos, hemorragias internas.

\section{Alterações Ósseas}

Uma evidência do papel das PGs na osteogênese, veio no início de 1980 a partir de observações feitas em crianças com doença cardíaca congênita, as quais foram tratadas com $\mathrm{PGE}_{1}$ para manter a patência do ducto arterioso, tendo sido observado proliferação óssea na superfície do periósteo como efeito colateral. Por outro lado, proliferação periosteal semelhante também foi observada em cães que receberam $\mathrm{PGE}_{1}$ para estudo de seus efeitos sobre o sistema cardiovascular (MILLER \& MARKS, 1994).

As PGEs possuem efeitos potentes sobre a reabsorção óssea in vitro e sobre a formação óssea, remodelação e modelação óssea in vivo. Quando administradas por via sistêmica, as PGEs possuem efeitos anabólicos potentes. Elas aumentam a taxa de formação óssea, modelação e remodelação, aumentam a formação óssea periosteal, assim como a massa óssea. As PGEs estimulam a replicação de osteoblastos e a formação óssea, conforme observado por MILLER \& MARKS (1994), sendo que as $\mathrm{PGE}_{1}$ e $\mathrm{PGE}_{2}$ aumentam a formação de tecido ósseo em cultura celular de crânio de rato, sugerindo que elas podem estimular células osteoprogenitoras.

A seqüência de eventos para o crescimento ósseo é a proliferação de células no periósteo, as quais mantêm a população de células precursoras de osteoblastos e suprem o osso central com osteoblastos (CHYUN et al., 1984).

$$
\text { TURNER \& COLLINS (1975) }
$$
demonstraram que crianças recém-nascidas cujas mães ingeriram salicilatos regularmente durante a gestação, apresentaram peso reduzido ao nascimento associado a aumento da taxa de mortalidade.

SLOTKIN et al. (1991), estudando o efeito dos glicocorticóides em fetos de rato, observaram que o tratamento pré-natal com dexametasona induzia a diminuição da proliferação celular em diversos tecidos, refletindo provavelmente uma ação seletiva destes medicamentos sobre certos tipos celulares e ainda 
sobre diversas fases da diferenciação celular, o que poderia ocasionar em retardo do crescimento pósnatal.

Já CHYUN et al. (1984) sugeriram que os corticóides parecem exercer efeito inibitório sobre as células precursoras de osteoblastos no periósteo, com conseqüente diminuição da formação óssea.

HANSEN et al. (1999), em estudo experimental em ratos, administraram dexametasona na dose de $0,8 \mathrm{mg} / \mathrm{kg}$, por via subcutânea, a fêmeas gestantes do $9^{\circ}$ ao $14^{\circ}$ dia de gestação. No vigésimo dia de gestação, estes animais foram submetidos a eutanásia e colhidos fragmentos maternos e fetais para análise. Os autores observaram que os fetos do grupo tratado com dexametasona apresentaram menor peso em comparação ao grupo não tratado, assim como alta taxa de mortalidade e fenda palatina.

Os glicocorticóides influenciam o metabolismo ósseo regulando o metabolismo do cálcio e do fosfato, exercendo efeito sobre a síntese de colágeno pelos osteoblastos e sobre degradação do colágeno pela colagenase, sendo que um dos principais efeitos indesejados desses agentes é a osteoporose (CHYUN et al. 1984).

\section{CONCLUSÕES}

É possível que a inibição da síntese de PGs durante a vida fetal, pelo uso de antiinflamatórios, possa provocar alterações na adaptação à vida extra-uterina, expondo o neonato a um risco cuja incidência é difícil de apreciar. Até a obtenção de maiores informações, na Medicina Veterinária, sobre os efeitos metabólicos, hematológicos e hemodinâmicos dos antiinflamatórios em fetos e recém nascidos, o uso destas drogas deve ser limitado a casos de extrema urgência e com controle por parte do profissional responsável. É importante que os clínicos tenham conhecimento da base farmacológica dos diferentes antiinflamatórios e seu mecanismo de ação (COX-1 ou COX-2) para que façam uma avaliação crítica prevendo os possíveis efeitos colaterais, principalmente durante a vida fetal.

Agentes inibidores da COX-2, enquanto preservam a COX-1, representam grande avanço no tratamento de várias patologias e podem ser indicados com maior segurança para tratamento de afecções das fêmeas domésticas.

\section{REFERÊNCIAS BIBLIOGRÁFICAS}

ADAMS, H.R. Prostaglandinas. In: BOOTH, N.H., Mc DONALD, L.E. Farmacologia e terapêutica em veterinária. 6.ed. Rio de Janeiro : Guanabara Koogan, 1992. p.363-369.
BAVOUX, F. Toxicité foetale des anti-inflammatoires non stérö̈diens. La Presse Medicale, v.21, n.40, p.1909-1912, 1992.

BAVOUX, F., BODIOU, C., CARLUS, C. Toxicité foetale des anti-inflammatoires non stérö̈diens. Archieves French Pediatrics, v.49, p.453-466, 1992.

CABROL, D., LANDESMAN, R., MULLER, J. et al. Treatment of polyhydramnios with prostaglandin syntetase inhibitor (indomethacin). American Journal Obstetric and Gynecology, v.157, p.422-426, 1987.

CHYUN, Y.S., KREAM, B.E., RAISZ, L.G. Cortisol decreases bone formation by inhibiting periosteal cell proliferation. Endocrinology, v.114, n.2, p.477-480, 1984.

DARMMANN, H.G. Preferential COX-2 inhibition: its clinical relevance for gastrointestinal non-steroidal anti-inflammatory rheumatic drug toxicity. Gastroenterology, v.37, n.1, p.4558,1999 .

DAVIES, P., MACINTYRE, D.E. Prostaglandins and inflamation. In: GALLIN, J.I., GOLDSTEIN, I.M., SNYDERMAN, R. Inflammation: basic principles and clinical correlates. 2.ed. New York : Raven, 1992. p.123138 .

DEKKER, G.A., SIBAI, B.M. Low-dose aspirin in the prevention of preeclampsia and fetal grouth retardation: Rationale mechanisms, and clinical trials. American Journal Obstetric Gynecology, v.168, p.214-227, 1993.

GOOTMAN, N., GOOTMAN, P.M. Perinatal cardiovascular function. New York : Marcel Dekker, 1983. Neural regulation of cardiovascular function in the perinatal period. p. 265 .

GORDON, M.G., SAMUELS, P. Indomethacin. Clinical Obstetrics and Gynecology, v.38, n.4, p.697-705, 1995.

GUYTON, A.C. Tratado de fisiologia médica. 8.ed. Rio de Janeiro : Guanabara Koogan, 1992. Fisiologia fetal e neonatal: p.818-825.

HANSEN, D.K., LABORDE, J.B., WALL, K.S., $\boldsymbol{e} \boldsymbol{t} \boldsymbol{a l}$. Pharmacokinetic considerations of dexamethasone-induced developmental toxicity in rats. Toxicology Science, v.48, n.2, p.230-239, 1999.

HICKOK, D.E., HOLLENBACH, K.A., REILLEY, S.F., $\boldsymbol{e}$ t al The association between decreased amniotic fuio volume and treatmennt with nonsteroidal anti-inflamatory agents for preterm labor. American Journal Obstetric Gynecology, v.160, p.1525-1531, 1989.

LOFFREDO, V., SLAOUI, R., BUSSIERE, P. Prescription d'indométacine dans les menances d'accouchement prématuré. Risque de cardiomyopathie. Presse Medicale, v.18, p.1124, 1989.

MANCHESTER, D., MARGOLIS, H.S., SHELDON, R.E Possible association between maternal indomethacin therapy and primary pulmonary hypertension of the newborn. American Journal Obstetric Gynecology, v.126, p.467469, 1976.

MILLER, S.C., MARKS, S.C. Effects of prostaglandins on the skeleton. Clinics in Plastic Surgery, v.21, p.393-398, 1994. 
OSTENSEN, M. Nonsteroidal anti-inflammatory drugs during pregnancy. Scandinavian Journal of Rheumatology Supplement, v.107, p.128-132, 1998.

REIN, A.J., NADJARI, M., ELCHALAL, U., et al. Contraction of the fetal ductus arteriosus induced by diclofenac. Case report. Fetal Diagnostics and Therapeutics, v.14, n.1, p.24$25,1999$.

REYES, J.L. Arachidonic acid metabolites and haemodynamics of the neonate. Pediatrics Nephrology, v.7, n.6, p.841-844, 1993.

RUDOLPH, A.M. The effects of non steroidal anti-inflamatory compounds on fetal circulation and pulmonary function. Obstetric Gynecology, v.58, p.635-665, 1981.

SCHROR, K. Aspirin and platelets: the antiplatelet action of aspirin and its role in thrombosis treatment and prophylaxis. Seminars of Thrombosis Homeostasis, v.23, n.4, p.349-356, 1997.
SIMON, L.S. Role and regulation of cyclooxygenase-2 during inflammation. American Journal of Medicine, v.106, n.5B p.37-42, 1999.

SLOTKIN, T.A., SEIDLER, F.J., KAVLOCK, R.J., et al. Fetal dexamethasone exposure impairs cellular development in neonatal rat heart and kidney: effects on DNA and protein in whole tissues. Teratology, v.43, p.301-306, 1991.

STUART, M.J., GROSS, S.J., ELRAD, H., et al. Effects of acetil salicylic acid ingestion on maternal and neonatal hemostasis. The New England Journal of Medicine, v.307, n.15, p. 909-912, 1982.

TURNER, G., COLLINS, E. Fetal effects of regular salicylate ingestion in pregnancy. The Lancet, v.23, p.338-339, 1975.

VIINIKA, L., HARTIKAINEN-SORRI, A.L., LUMME, R, et al Low dose aspirin in hypertensive pregnant women: effect on pregnancy outcome and prostacyclin-thromboxane balance in mother and newborn. Brazilian Journal Obstetrics Gynaecology, v.100, n.9, p.809-815, 1993. 